\title{
Star-formation rate in compact star-forming galaxies
}

\author{
I. Y. Izotova • Y. I. Izotov
}

(C) Springer-Verlag $\bullet \bullet \bullet \bullet$

\begin{abstract}
We use the data for the $\mathrm{H} \beta$ emission-line, far-ultraviolet (FUV) and mid-infrared $22 \mu \mathrm{m}$ continuum luminosities to estimate star formation rates $<$ SFR $>$ averaged over the galaxy lifetime for a sample of about 14000 bursting compact star-forming galaxies (CSFGs) selected from the Data Release 12 (DR12) of the Sloan Digital Sky Survey (SDSS). The average coefficient linking $<\mathrm{SFR}>$ and the star formation rate $\mathrm{SFR}_{0}$ derived from the $\mathrm{H} \beta$ luminosity at zero starburst age is found to be 0.04 . We compare $\langle\mathrm{SFR}>\mathrm{s}$ with some commonly used SFRs which are derived adopting a continuous star formation during a period of $\sim$ $100 \mathrm{Myr}$, and find that the latter ones are $2-3$ times higher. It is shown that the relations between SFRs derived using a geometric mean of two star-formation indicators in the UV and IR ranges and reduced to zero starburst age have considerably lower dispersion compared to those with single star-formation indicators. We suggest that our relations for $<\mathrm{SFR}>$ determination are more appropriate for CSFGs because they take into account a proper temporal evolution of their luminosities. On the other hand, we show that commonly used SFR relations can be applied for approximate estimation within a factor of $\sim 2$ of the $<$ SFR $>$ averaged over the lifetime of the bursting compact galaxy.
\end{abstract}

\section{Y. Izotova}

Astronomical Observatory of Taras Shevchenko Kyiv National University

Observatorna str., 3, 04053, Kyiv, Ukraine

tel: +380444860021 , fax: +380444862191

e-mail:izotova@observ.univ.kiev.ua

Y. I. Izotov

Main Astronomical Observatory of the National Academy of Sciences of Ukraine

Zabolotnoho str., 27, 03143, Kyiv, Ukraine

tel: +380445264771 , fax: +380445262197

e-mail:izotov@mao.kiev.ua
Keywords Galaxies: dwarf - Galaxies: fundamental parameters - Galaxies: starburst — Galaxies: star formation

\section{Introduction}

The star formation rate (SFR) is one of the most important parameters regulating the galaxy evolution. Many studies have been made in the past to derive SFRs from the luminosities at various wavelengths, spanning from the far-UV (FUV), where the massive stars emit the bulk of their energy, to the infrared, where the dustreprocessed light from those stars emerges, and to the radio, which is a tracer of supernova activity at wavelengths $\lambda \gtrsim 20 \mathrm{~cm}$, while free-free emission from $\mathrm{H}$ II regions dominates at wavelengths shorter than $6 \mathrm{~cm}$.

Various luminosities, which serve as SFR monochromatic indicators, have been considered by e.g. Kennicutt (1998) and Calzetti et al. (2010), while combinations of the luminosities at two or more different wavelengths have been analysed in other studies (e.g. Kennicutt et al. 2009; Hao et al. 2011; Lee et al. 2013; Boquien et al. 2016; Puglisi et al. 2016). The luminosities of recombination $\mathrm{H} \alpha, \mathrm{H} \beta$ and other hydrogen lines, UV, mid-infrared (MIR), far-infrared (FIR) and radio continuum luminosities are among the most commonly used indicators. Emission at these various wavelengths is generated by stars with different lifetimes, with the shortest lifetimes for the most massive stars producing hydrogen recombination lines. These SFR indicators have been applied to different samples of SF galaxies at redshifts $z$ from 0 to $\sim 6$ (Reddv et al. 2010, 2015; Curtis-Lake et al. 2013; Jiang et al. 2013; Kashino et al. 2013; Battisti et al. 2015; Panella et al. 2015; Shivaei et al. 2015, 2016; Duarte Puertas et al. 2017; Karman et al. 2017) and for samples with various physical properties. 

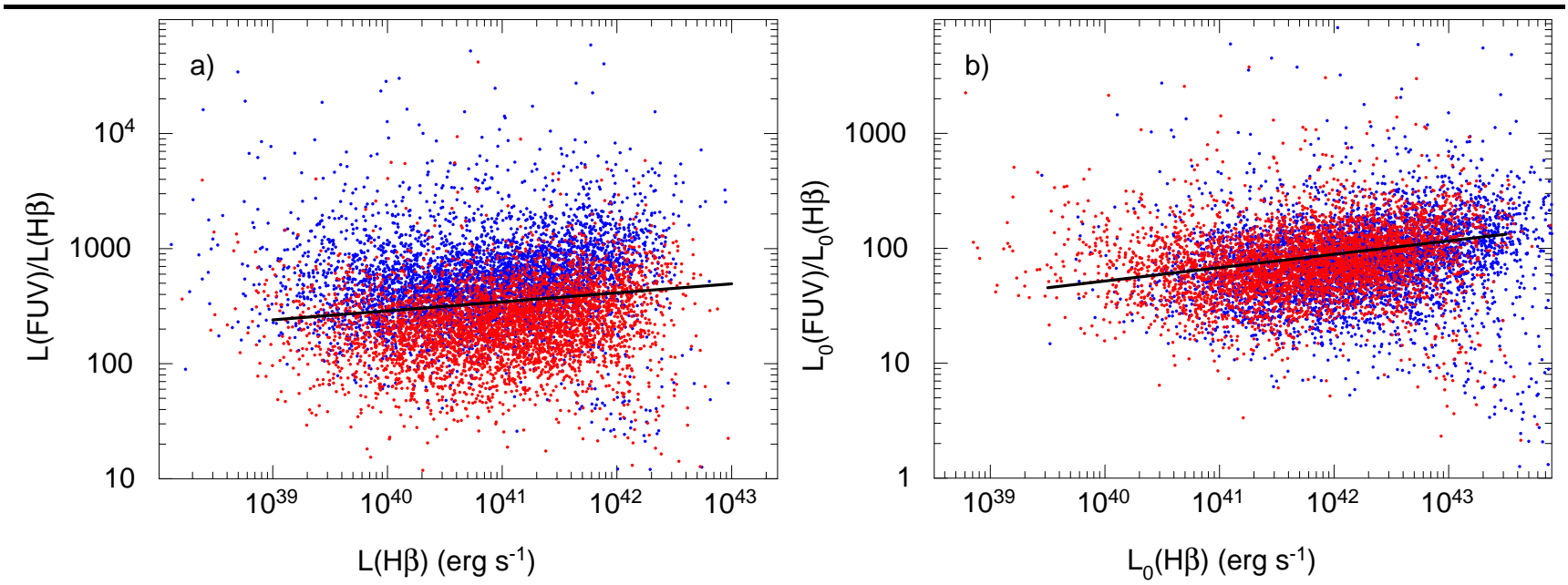

Fig. 1 a) The relation between the FUV-to-H $\beta$ luminosity ratio and the $\mathrm{H} \beta$ luminosity. CSFGs with $\mathrm{EW}(\mathrm{H} \beta) \geq 50 \AA$ and $\mathrm{EW}(\mathrm{H} \beta)<50 \AA$ are shown by red and blue symbols, respectively. The solid line is the maximum-likelihood relation $\log \left[\nu L_{\nu}(\mathrm{FUV}) / L(\mathrm{H} \beta)\right]=0.078 \times \log L(\mathrm{H} \beta)-0.68$ for the entire sample. b) Same as in a), but luminosities are reduced to a zero starburst age. The solid line is the maximum-likelihood relation $\log \left[\nu L_{\nu, 0}(\mathrm{FUV}) / L_{0}(\mathrm{H} \beta)\right]=0.12 \times \log L_{0}(\mathrm{H} \beta)-2.94$ for the entire sample. $\mathrm{H} \beta$ luminosities in both panels are expressed in erg s${ }^{-1}$

SFR calibrations are affected by many input factors (discussed e.g. by Zhang et al. 2013). The initial mass function (IMF), metallicity, binary interactions, evolution models of rotating and non-rotating stars and stellar atmosphere models are among them. Some of these factors are not well known and various assumptions on them may lead to SFR variations of $\sim 0.3 \mathrm{dex}$. One of the important factors influencing SFR is the star formation history.

The SFR calibrations are usually obtained from population synthesis models adopting an IMF and prolonged star-formation activity. In particular, several widely used calibrations (e.g. Kennicutt 1998; Calzetti et al. 2010) are obtained assuming the Salpeter (1955) IMF and relatively large period of $\sim 100 \mathrm{Myr}$ for star formation with a constant rate. With this relatively long period, the luminosities in different wavelength ranges and their ratios are slowly changing with time. Besides that some other SF histories, e.g. with exponentially decaying or rising SFRs, were also considered in some papers.

However, such an assumption may not be valid in the case of compact star-forming galaxies (CSFGs) with star-formation proceeded in short starbursts, which dominate in the galaxy light, but quickly decay on the time scales of $\lesssim 10$ Myr. Indeed, Starburst99 models (Leitherer et al. 1999, 2014) for instantaneous bursts predict that the $\mathrm{H} \beta$ luminosity decreases by two orders of magnitude during the first $10 \mathrm{Myr}$. Therefore, the definition of the SFR for starburst galaxies is somewhat uncertain because of the uncertainties in the determination of the period of star formation. Because of this the use of the SFR calibrations discussed above for starburst galaxies and the comparison of their SFRs with those for star-forming galaxies with prolonged star formation are questionable.

In this paper we propose a recipe for the determination of the SFR averaged over the lifetime of the bursting compact galaxies and discuss the applicability of commonly used SFR calibrations for CSFGs with strong emission lines where star-formation is occurred in short intense starbursts. Izotov et al. (2016a) have constructed this CSFGs sample from the Sloan Digital Sky Survey (SDSS). Selection criteria for the sample and its general characteristics are considered in Sect. 2, We discuss the star formation history in CSFGs in Sect. 3. The relations for the SFR determination at a zero starburst age are considered in Sect. 4 In Sect. 5 the derived SFRs averaged over the CSFG lifetime are compared with commonly used SFR calibrations. We summarize our results in Sect. 6.

\section{The sample}

Izotov et al. (2016a) have constructed a sample of 14000 CSFGs from the spectroscopic data base of the SDSS Data Release 12 (DR12) (Alam et al. 2015) which includes classic SDSS subsample from Data Releases 9 and earlier releases and BOSS subsample from Data Releases 10 and 12. The spectra of these two different subsamples were obtained with two different round spectroscopic apertures, 3 arcsec and 2 arcsec in diameter, respectively. Furthermore, spectra from 

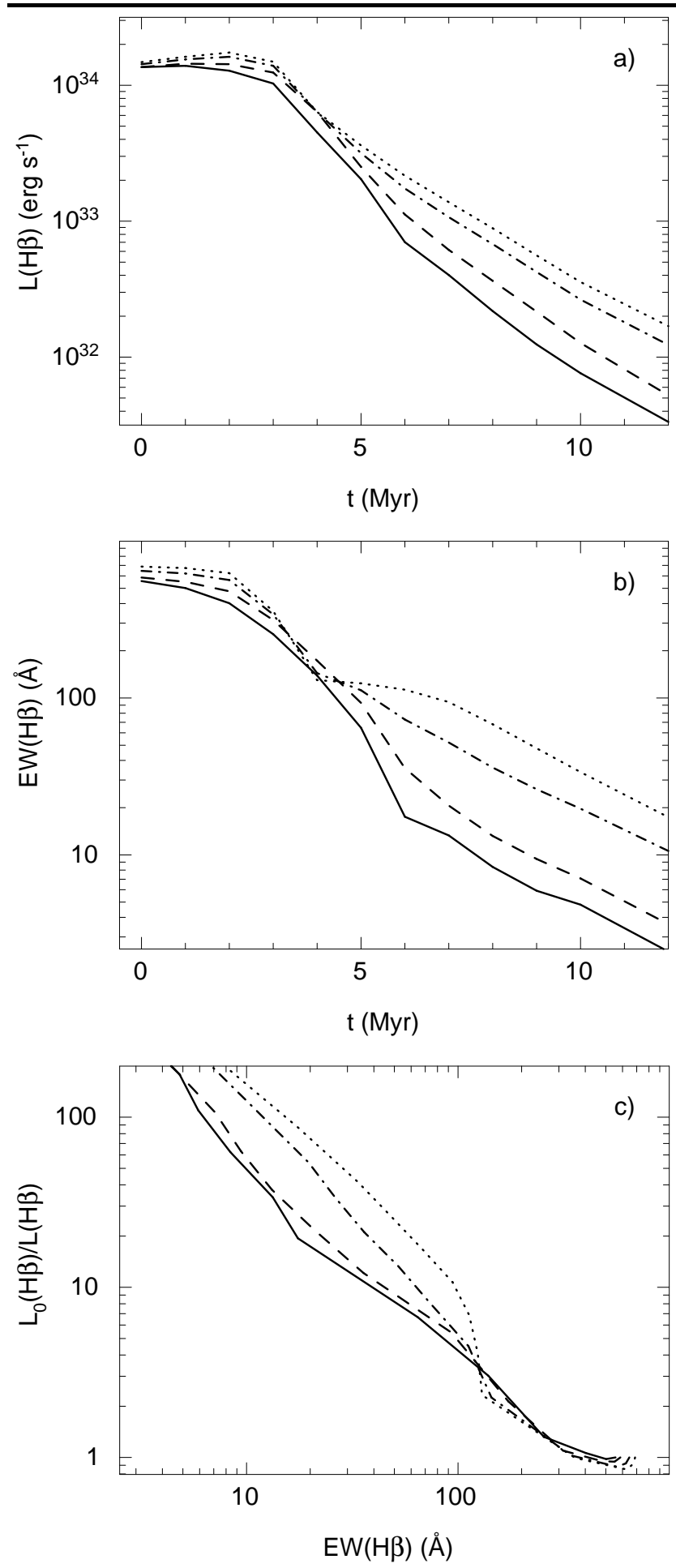

Fig. 2 a) The dependences of the $\mathrm{H} \beta$ luminosity on the starburst age for instantaneous burst models with various metallicities: 1/50 solar (dotted line), 1/20 solar (dashdotted line), $1 / 5$ (dashed line), and 1/2.5 solar (solid line) (Leitherer et al. 1999). Luminosities are derived for a starburst with a mass of $1 \mathrm{M}_{\odot}$. b) Same as in a) but for the relation between the $\mathrm{H} \beta$ equivalent width $\mathrm{EW}(\mathrm{H} \beta)$ and the starburst age. c) The dependences of the $\mathrm{H} \beta$ luminosity correction factor on the $\mathrm{H} \beta$ equivalent width the classic SDSS and BOSS subsamples cover different wavelength ranges, $\sim 3800-9200 \AA$ and $\sim 3600-$ $10000 \AA$, respectively.

The objects were selected by their compactness adopting the Petrosian radius $R_{50} \lesssim 3$ arcsec to minimize aperture corrections in the comparison of spectroscopic and photometric data at various wavelengths from UV to mid-infrared, by strong emission lines as defined by the $\mathrm{H} \beta$ equivalent width $\mathrm{EW}(\mathrm{H} \beta) \gtrsim 10 \AA$, and by absence of AGN spectral features. CSFGs are distributed over the redshift range $z=0-1$ with a median value of 0.2 . The median value of $\mathrm{EW}(\mathrm{H} \beta)$ for the sample is $\sim 40 \AA$, while a considerable fraction of CSFGs $(\sim 10$ percent) is characterized by much higher $\mathrm{EW}(\mathrm{H} \beta)>100 \AA$. We note that strong $\mathrm{H} \delta, \mathrm{H} \gamma$ and $\mathrm{H} \beta$ emission lines are seen in spectra of all galaxies from our sample, regardless of the redshift. As for $\mathrm{H} \alpha$, it is present in spectra of galaxies with $z<0.4$ from the classic SDSS subsample and with $z<0.52$ from the BOSS subsample. The presence of several hydrogen lines allows the reliable simultaneous determination of extinction and underlying stellar absorption from the observed Balmer decrement, following to Izotov et al. (1994). Correspondingly, fluxes of hydrogen lines were corrected for both effects. In general, correction for underlying absorption in CSFGs is small, not exceeding $\sim 10$ percent on average for the $\mathrm{H} \beta$ emission line.

We use the method described by Izotov et al. (2016a) to derive stellar masses of CSFGs from SED fitting of the SDSS spectra. The method includes both the stellar and nebular continua and is outlined below. The starformation history is approximated assuming a short burst with age $t_{\text {young }}<10 \mathrm{Myr}$ and a continuous star formation with a constant SFR for the older population with a constant SFR during the time interval between $t_{i}$ and $t_{f}\left(t_{f}<t_{i}\right.$ and zero age is now $)$. Thus, the age of the oldest stars in the model is $t_{\text {old }} \equiv t_{i}$. The contribution of each stellar population to the SED was parameterized by the ratio $b=M_{\text {young }} / M_{\text {old }}$, where $M_{\text {young }}$ and $M_{\text {old }}$ are the masses of the young and old stellar populations formed during the recent burst with $t_{\text {young }}<10 \mathrm{Myr}$ and the prior continuous star formation between $t_{f}$ and $t_{i}$, respectively.

We note that star formation rate in CSFGs is uncertain at large ages and may not be constant. Weisz et al. (2016) analyzed resolved stellar populations of dwarf galaxies of various morphological types in the Local Volume and concluded that the star formation histories (SFHs) are complex and the mean values are inconsistent with simple SFH models, e.g., single bursts, constant SFRs, or smooth, exponentially declining SFRs. However, results by Weisz et al. (2016) are obtained for low-mass and relatively quiescent galaxies, while our 
CSFGs are more massive and experience vigorous recent SF episode. Furthermore, emission in the optical SDSS spectra used for SED fitting is strongly dominated by the young stellar population. This makes the stellar mass determination of the old stellar population even more uncertain. In spite of these uncertainties we adopted the simplest SFH with a constant SFR for the old stellar population and rely mainly on the statistical properties of the sample.

To fit the SED we carried out a series of 5000 Monte Carlo simulations for each galaxy by randomly varying $t_{\mathrm{y}}, t_{i}, t_{f}$, and $b$. A grid of instantaneous burst SEDs with a wide range of ages from $0 \mathrm{Myr}$ to $15 \mathrm{Gyr}$ and various metallicities was calculated with Starburst99 (Leitherer et al. 1999, 2014) to derive the SED of the galaxy stellar component. Input parameters for the grid calculations included Padova stellar evolution tracks (Girardi et al. 2000), models of stellar atmospheres by Lejeune et al. (1997) and Schmutz et al. (1992), and the stellar initial mass function of Salpeter (1955) with the upper and low stellar mass limits of $100 \mathrm{M}_{\odot}$ and $0.1 \mathrm{M}_{\odot}$, respectively. Then the stellar SED with any star-formation history can be obtained by integrating the instantaneous burst SEDs over time with a specified time-varying SFR.

Given the electron temperature $T_{\mathrm{e}}$ in the $\mathrm{H}$ II region, we interpolated emissivities by Aller (1984) for the nebular continuum in the Te range of $5000-20000 \mathrm{~K}$. Then the nebular continuum luminosity at any wavelength is derived from the observed $\mathrm{H} \beta$ luminosity. We also calculate the equivalent width $\mathrm{EW}(\mathrm{H} \beta)$ for each model, integrating over the time luminosities of emission lines and adjacent continua.

The best solution is required to fulfill the following conditions. First, only models, in which $\operatorname{EW}(\mathrm{H} \beta)$ is in agreement with the observed value within 10 percent, were selected. Second, the best modelled SED satisfying first condition was found from $\chi^{2}$ minimization of the deviation between the modelled and the observed continuum.

Comparing different sets of Monte Carlo realizations for the same galaxy we find that the dispersion of the derived stellar masses is less than 0.1 dex. In our calculations we do not take into account the stellar mass returned to the interstellar medium via mass loss. It is estimated to be less than $10-15 \%$, resulting in the lower stellar mass by the same amount. However, we neglect this small reduction of the stellar mass because of uncertainties in its determination.

The distribution of CSFG stellar masses is peaked at $M_{\star} \sim 10^{9} \mathrm{M}_{\odot}$, while the distribution of $\mathrm{H} \beta$ luminosities is peaked at $L(\mathrm{H} \beta) \sim 10^{41} \mathrm{erg} \mathrm{s}^{-1}$, corresponding to $\mathrm{SFR} \sim 1 \mathrm{M}_{\odot} \mathrm{yr}^{-1}$ as derived from the Kennicutt
(1998) relation. Thus, on average, the time of only $\sim$ 1 Gyr is needed to build up the mass of the CSFG with the SFR derived from the $\mathrm{H} \beta$ luminosity. This is an indication of the bursting nature of star formation in these galaxies. Furthermore, since the $\mathrm{H} \beta$ luminosity in CSFGs is nearly proportional to their stellar mass, this conclusion holds for CSFG with any stellar mass. On the other hand, according to SED fitting, some fraction of stars with ages older than 1 Gyr is present in most CSFGs. Therefore, an average SFR in bursting galaxy may be lower than that derived from the observed $\mathrm{H} \beta$ luminosity with the Kennicutt (1998) relation.

The SDSS spectroscopic data are supplemented by the photometric data obtained with the Galaxy Evolution Explorer (GALEX) in the UV range and the Wide-field Infrared Survey Explorer (WISE) in the MIR range. We note that nearly 70 percent of CSFGs were detected by both the GALEX and WISE. The SDSS spectra are used in this paper to derive galaxy stellar masses $M_{\star}, \mathrm{H} \beta$ luminosities $L(\mathrm{H} \beta)$, star formation rates $\operatorname{SFR}(\mathrm{H} \beta)$, and equivalent widths $\operatorname{EW}(\mathrm{H} \beta)$, while GALEX and WISE data are used for the determination of FUV and $22 \mu \mathrm{m}$ luminosities, SFR(FUV) and $\operatorname{SFR}(22 \mu \mathrm{m})$.

The luminosities are derived adopting distance obtained from the NED cosmological calculator Wright 2006) for the cosmological parameters $H_{0}=67.1 \mathrm{~km}$ $\mathrm{s}^{-1} \mathrm{Mpc}^{-1}, \Omega_{m}=0.318, \Omega_{\Lambda}=0.682$ (Planck Collaboration XVI 2014) and a flat geometry. The correction of FUV and $\mathrm{H} \beta$ luminosities for extinction was done it two steps. Firstly, they were corrected for the Milky Way extinction at the observed wavelengths adopting $A(V)_{\mathrm{MW}}$ from the NED. Secondly, correction for the internal galaxy extinction derived from the hydrogen Balmer decrement in the SDSS spectra after its correction for the Milky Way extinction was applied at the restframe wavelengths. In all cases a reddening law by Cardelli et al. (1989) and a total-to-selective extinction ratio $R(V)=3.1$ were adopted. Finally, $\mathrm{H} \beta$ luminosities were corrected for the spectroscopic aperture by using the correction factor of $2.512^{r(\mathrm{ap})-r}$, where $r$ and $r$ (ap) are the total SDSS magnitude and the magnitude within the spectroscopic aperture of 3 arcsec for classic SDSS objects and of 2 arcsec for BOSS objects.

\section{Star formation history in CSFGs}

The comparison of the ultraviolet-to- $\mathrm{H} \beta$ luminosity ratios in galaxies with different equivalent widths $\mathrm{EW}(\mathrm{H} \beta)$ of the $\mathrm{H} \beta$ emission line has demonstrated that star formation in CSFGs is bursting in nature (Izotov et al. 2016a). To illustrate this we show in 

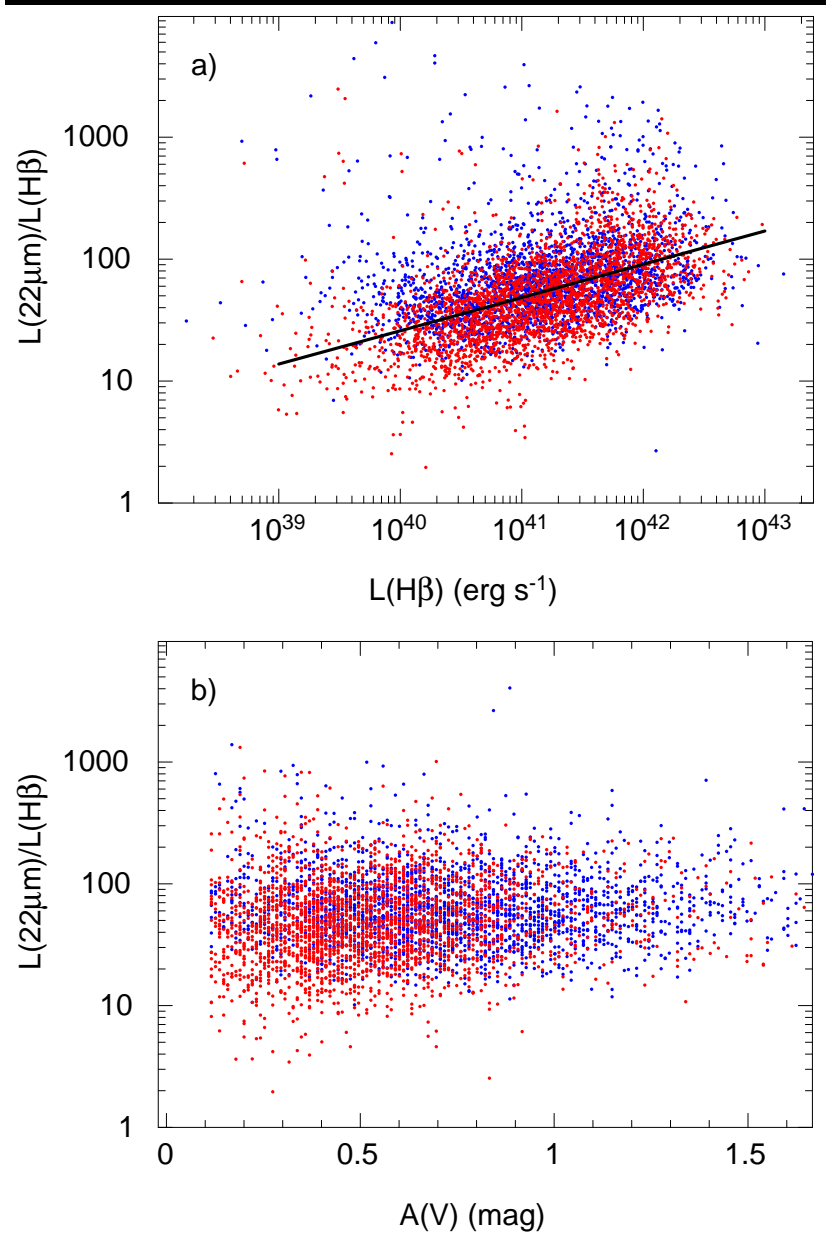

Fig. 3 a) The relation between the $22 \mu \mathrm{m}$-to-H $\beta$ luminosity ratio and the $\mathrm{H} \beta$ luminosity. CSFGs with $\mathrm{EW}(\mathrm{H} \beta) \geq$ $50 \AA$ and $\mathrm{EW}(\mathrm{H} \beta)<50 \AA$ are shown by red and blue symbols, respectively. The solid line is the maximum-likelihood relation $\log \left[\nu L_{\nu}(22 \mu \mathrm{m}) / L(\mathrm{H} \beta)\right]=0.35 \times \log L(\mathrm{H} \beta)-9.50$ for the entire sample. The $\mathrm{H} \beta$ luminosities are expressed in $\mathrm{erg} \mathrm{s}^{-1}$. b) The dependence of the $22 \mu \mathrm{m}$-to- $\mathrm{H} \beta$ luminosity ratio on the extinction $A(V)$

Fig. 17 the dependence of the FUV-to-H $\beta$ luminosity ratio on the $\mathrm{H} \beta$ luminosity for CSFGs. It is seen that there is an offset between CSFGs with high and low $\mathrm{EW}(\mathrm{H} \beta)_{\mathrm{s}}$ (red and blue symbols, respectively). Izotov et al. (2016a) assumed that this difference is due to that the star formation in CSFGs occurred in short starbursts. This assumption is further supported by the fact that UV emission in these galaxies is mainly produced in one bright compact $\mathrm{SF}$ region as it is evidenced by the near-UV (NUV) acquisition images of some CSFGs obtained with the Cosmic Origins Spectrograph (COS) onboard of the Hubble Space Telescope (HST) (Izotov et al. 2016b, 2018; Yang et al. 2017).

In this paper, according to Izotov et al. (2016a) we adopt that youngest stellar population in CSFGs with
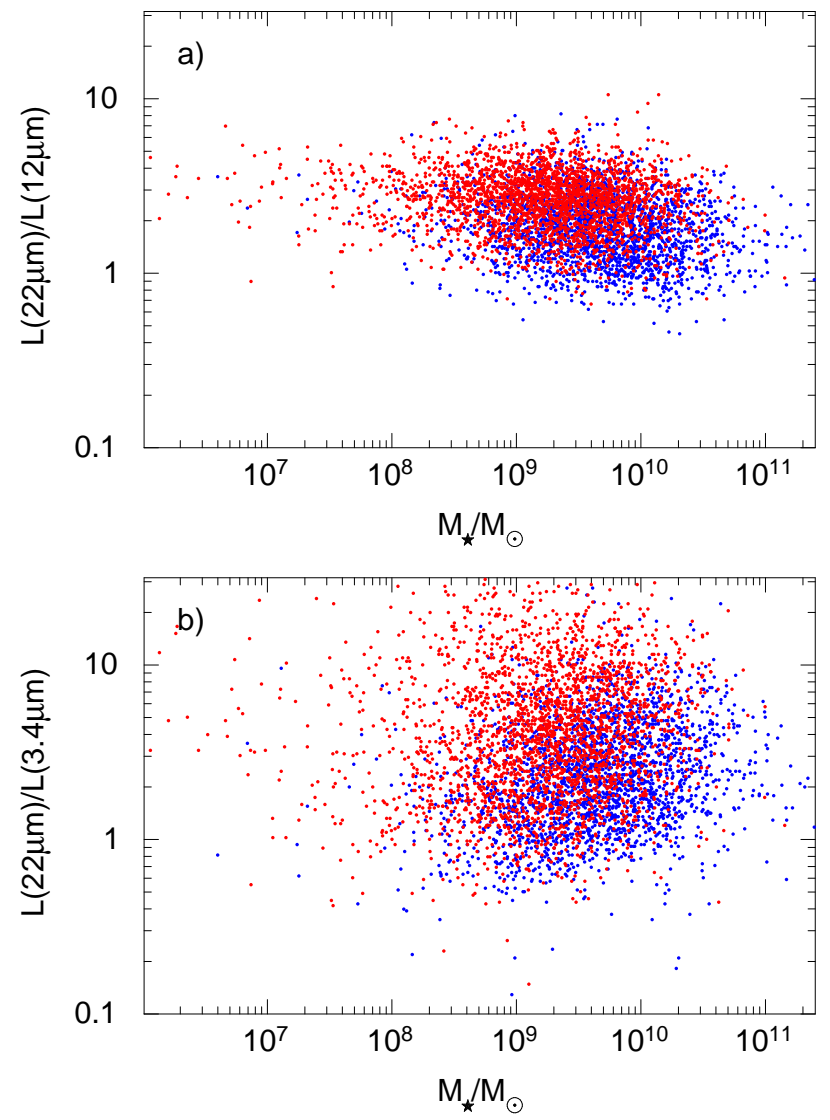

Fig. 4 a) The relation between the $22 \mu \mathrm{m}$-to- $12 \mu \mathrm{m}$ luminosity ratio and the stellar mass. b) The dependence of the $22 \mu \mathrm{m}$-to- $3.4 \mu \mathrm{m}$ luminosity ratio on the stellar mass. CSFGs with $\mathrm{EW}(\mathrm{H} \beta) \geq 50 \AA$ and $\mathrm{EW}(\mathrm{H} \beta)<50 \AA$ are shown by red and blue symbols, respectively

an age of a few Myr is formed in instantaneous bursts. In this case both the $\mathrm{H} \beta$ luminosity, which is produced by ionizing radiation of the most-massive stars, and the $\mathrm{EW}(\mathrm{H} \beta)$ rapidly decrease by more than two orders of magnitude on a time scale of $10 \mathrm{Myr}$ (Fig. 2a,b; Leitherer et al. 1999). We note that there is a dependence of relations in Fig. 2 on the metallicity. Similarly, the FUV luminosity also decreases, but more slowly, because it is produced by less massive and thus more long-lived stars. Izotov et al. (2016a) proposed the following correction to reduce the observed $\mathrm{H} \beta$ luminosity to the luminosity at a zero starburst age for a metallicity of $1 / 5$ solar (dashed line in Fig. 2r) corresponding to typical metallicities of CSFGs from our sample:

$\Delta \log L(\mathrm{H} \beta)=2.7-\log [\mathrm{EW}(\mathrm{H} \beta)]$,

for $\log \mathrm{EW}(\mathrm{H} \beta) \leq 2.7$, otherwise $\Delta \log L(\mathrm{H} \beta)=0$. Similarly,

$\Delta \log L(\mathrm{FUV})=0.39 \times \Delta \log L(\mathrm{H} \beta)$. 

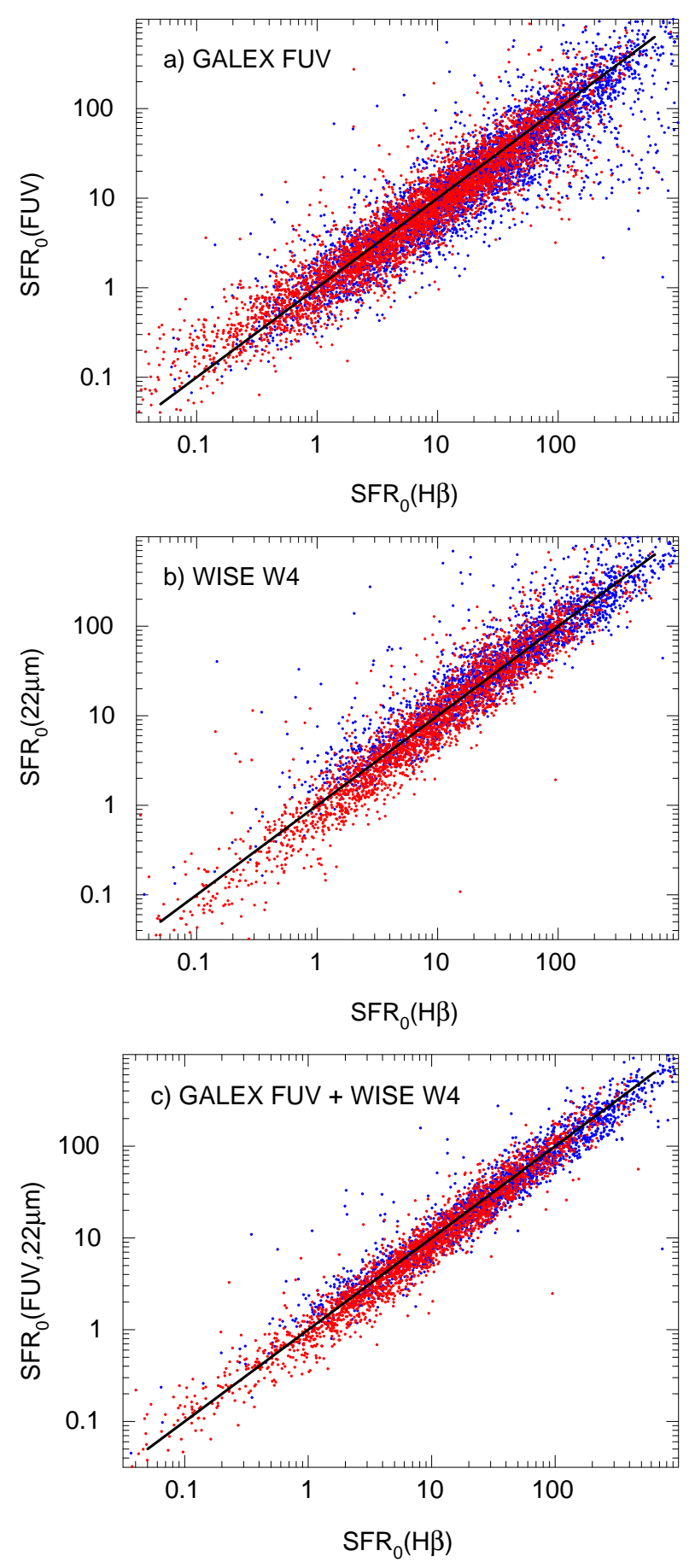

Fig. 5 Relations between the star formation rate $\mathrm{SFR}_{0}(\mathrm{H} \beta)$ (Eq. 3) and a) the star formation rate $\mathrm{SFR}_{0}$ (FUV) (Eq. [5), b) the star formation rate $\mathrm{SFR}_{0}(22 \mu \mathrm{m})$ (Eq. 6) and c) the star formation rate $\mathrm{SFR}_{0}(\mathrm{FUV}, 22 \mu \mathrm{m})$ (Eq. 7).All SFRs are expressed in $\mathrm{M}_{\odot} \mathrm{yr}^{-1}$. They are derived from luminosities corrected for the starburst age. CSFGs with $\operatorname{EW}(\mathrm{H} \beta) \geq 50 \AA$ and $\mathrm{EW}(\mathrm{H} \beta)<50 \AA$ are shown by red and blue symbols, respectively. Solid lines in all panels are one by one relations
However, we do not apply corrections Eqs. 1 and 2 to the data for CSFGs. Instead we use cubic spline fits of the relation in Fig. 2 c for the $\mathrm{H} \beta$ luminosity and corresponding fits for the FUV luminosity (not shown) to apply luminosity corrections for the respective metallicity of the galaxy. We find that there is no difference between the FUV-to-H $\beta$ luminosity ratios reduced to a zero starburst age for the galaxies with high observed $\mathrm{EW}(\mathrm{H} \beta) \mathrm{s}$ (red symbols in Fig. 10) and the galaxies with low observed $\mathrm{EW}(\mathrm{H} \beta) \mathrm{s}$ (blue symbols in Fig. 1 b). The dispersion of data in Fig. 10 is also considerably reduced as compared to that in Fig. 17a.

In Fig. 3a for our sample we show the relation of the WISE $22 \mu \mathrm{m}$-to- $\mathrm{H} \beta$ luminosity ratio on the apertureand extinction-corrected $\mathrm{H} \beta$ luminosity. The presence of a tight correlation between the $22 \mu \mathrm{m}$ and $\mathrm{H} \beta$ luminosities (Fig. 3a, Izotov et al. 2014) allows us to use the infrared luminosity for the determination of SFR despite the fact that only a fraction of the UV radiation is absorbed by dust and re-emitted in the IR range. It is seen that this relation is nonlinear because the $L(22 \mu \mathrm{m}) / L(\mathrm{H} \beta)$ ratio increases with $L(\mathrm{H} \beta)$. This finding is in accord with many previous studies (e.g. Calzetti et al. 2010; Izotov et al. 2014) and may be attributed to the presence of warmer dust in galaxies with the higher $L(\mathrm{H} \beta)$, in agreement with predictions of Draine \& Li (2007).

At variance with $\mathrm{FUV}$-to- $\mathrm{H} \beta$ luminosity ratios shown in Fig. 17a, there is no difference between the galaxies with high and low $\mathrm{EW}(\mathrm{H} \beta) \mathrm{s}$ in Fig. 3 a. Since $22 \mu \mathrm{m}$ emission is produced by dust, the relation in Fig. 3 a implies that dust in CSFGs is mainly heated by the same stellar population which produces $\mathrm{H} \beta$ emission, in accord with conclusion made by Izotov et al. (2014). This conclusion is further supported by the absence of any dependence of the $L(22 \mu \mathrm{m}) / L(\mathrm{H} \beta)$ ratio on extinction in $A(V)$, shown in Fig. 3 $\mathrm{b}$. In addition, the distribution in Fig. 3b suggests that there is no need to correct $L(22 \mu \mathrm{m})$ for reddening, particularly due to low extinction in the optical range.

The absence of an offset between distributions of CSFGs with low and high $\mathrm{EW}(\mathrm{H} \beta)$ (Fig. 3a) implies that reduction of the $22 \mu \mathrm{m}$ luminosity to a zero starburst age should be done in the same way as that for the $\mathrm{H} \beta$ luminosity, i.e. using Eq. 1. This is in contrast with the results of many other papers where it has been concluded that infrared emission in normal star-forming galaxies is produced by a population continuously formed over a period $\gtrsim 100 \mathrm{Myr}$ (e.g. Calzetti et al. 2010).

We note that the WISE $12 \mu \mathrm{m}$ luminosity can also be used as a SFR indicator. The $L(22 \mu \mathrm{m}) / L(12 \mu \mathrm{m})$ ratio is shown in Fig. 4 a as a function of the stellar mass of the galaxy $M_{\star}$. It is seen that this ra- 
tio is fairly constant with small dispersion of individual CSFGs, indicating a tight correlation between the two luminosities. The $12 \mu \mathrm{m}$ band at variance with the $22 \mu \mathrm{m}$ band includes emission of polycyclic aromatic hydrocarbons (PAH) which can be heated by both starforming regions and older stellar populations. However, Wu et al. (2006) have shown that PAH emission is absent in the most metal-poor blue compact dwarf (BCD) galaxies and its strength is generally suppressed in a low-metallicity environment. Our CSFGs in this respect are similar to BCDs by having high-excitation $\mathrm{H}$ II regions and low-metallicity. Furthermore, Hunt et al. (2010) showed that the fraction of PAH emission normalized to the total infrared (IR) luminosity is considerably smaller in metal-poor BCDs $(\sim 0.5 \%)$ than that in metal-rich SFGs $(\sim 10 \%)$.

Contrary to that the WISE emission at $3.4 \mu \mathrm{m}$ is dominated by the light of cool stars and does not correlate with $22 \mu \mathrm{m}$ (Fig. 4b). Therefore, $3.4 \mu \mathrm{m}$ luminosity is not good SFR indicator.

\section{Star formation rates with luminosities reduced to zero starburst age}

One of the aims of this paper is to find consistent relations for SFRs derived from emission in the $\mathrm{UV}, \mathrm{H} \beta$ and infrared ranges for the sample of CSFGs. To achieve this we first reduce all luminosities to a zero starburst age by using equations in Sect. 3. Second, we derive the star formation rate $\mathrm{SFR}_{0}(\mathrm{H} \beta)$ from the $\mathrm{H} \beta$ luminosity at a zero starburst age adopting $L(\mathrm{H} \alpha)=2.8 \times L(\mathrm{H} \beta)$ and using the relation of Kennicutt (1998):

$\log \mathrm{SFR}_{0}(\mathrm{H} \beta)=\log L_{0}(\mathrm{H} \beta)-40.655$,

where $\log L_{0}(\mathrm{H} \beta)=\log L(\mathrm{H} \beta)+\Delta \log L(\mathrm{H} \beta), \mathrm{SFR}_{0}$ is in $\mathrm{M}_{\odot} \mathrm{yr}^{-1}$ and luminosities are in erg $\mathrm{s}^{-1}$. The $\mathrm{SFR}_{0}(\mathrm{H} \beta)$ is linked to the mass of the young stellar population $M_{\text {young }}$, formed in the burst, by a simple relation

$\operatorname{SFR}_{0}(\mathrm{H} \beta) \sim 2.8 \times 10^{-7} \frac{M_{\text {young }}}{M_{\odot}}$.

Here we use the relation Eq. 3 and the ratio of $\sim 10^{34.1}$ of the $\mathrm{H} \beta$ luminosity at zero age to the stellar mass of a single stellar population by Leitherer et al. (1999) which is only weakly dependent on metallicity.

Third, we adopt that $\mathrm{SFR}_{0}(\mathrm{H} \beta), \mathrm{SFR}_{0}(\mathrm{FUV})$, and $\operatorname{SFR}_{0}(22 \mu \mathrm{m})$ are equal at a zero starburst age. From these assumptions and using relations between luminosities at respective wavelengths (Izotov et al. 2014), we find

$\log \mathrm{SFR}_{0}(\mathrm{FUV})=0.84 \log L_{0}(\mathrm{FUV})-36.02$, $\log \mathrm{SFR}_{0}(22 \mu m)=0.79 \log L_{0}(22 \mu m)-33.50$

where $\mathrm{SFR}_{0} \mathrm{~S}$ are in $\mathrm{M}_{\odot} \mathrm{yr}^{-1}$ and luminosities are in $\operatorname{erg~} \mathrm{s}^{-1}$.

Furthermore, we introduce a combination of starformation indicators in the FUV and $22 \mu \mathrm{m}$ ranges, which can be defined as a geometric mean of SFRs in these two wavelength ranges:

$$
\begin{aligned}
\log \mathrm{SFR}_{0}(\mathrm{FUV}, 22 \mu \mathrm{m})= & 0.5\left[\log \mathrm{SFR}_{0}(\mathrm{FUV})\right. \\
& \left.+\log \mathrm{SFR}_{0}(22 \mu \mathrm{m})\right] .
\end{aligned}
$$

In Fig. 5 we show the relations between $\mathrm{SFR}_{0}$ 's derived from monochromatic and combined SFR indicators. It is seen that all these relations do not deviate from the one by one relations, although the dispersions of points are different. The largest dispersion is found for the relation between $\mathrm{SFR}_{0} \mathrm{~s}$ derived from the $\mathrm{H} \beta$ and FUV luminosities (Fig. 5a), while it is somewhat lower if infrared indicators are used (Figs. 5bc). It is also seen that there are no offsets between the galaxies with low and high observed $\mathrm{EW}(\mathrm{H} \beta) \mathrm{s}$ (respectively blue and red dots in Fig. 5). The geometric mean with the lowest dispersion of data is likely the most appropriate to use in the case of the CSFG sample (Fig. 56).

\section{Comparison with star formation rate calibrations by other authors}

In this Section we compare our SFRs with the ones derived by other authors from the luminosities at the same wavelengths. The purpose of this comparison is to understand whether can commonly used SFR calibrations be applied to derive reasonable SFRs in bursting galaxies averaged over their lifetime. Although those authors obtained their relations for SFGs selected without restrictions on the galaxy morphology, we apply their relations to our CSFRs. We remind that advantage of our sample is that only relatively modest corrections for aperture are needed for our compact galaxies when observations with different telescopes and different apertures are compared. This correction, e.g. of the $\mathrm{H} \beta$ flux derived from the SDSS spectrum, for extended galaxies can be large and uncertain. Furthermore, the comparison of SFRs is complicated by the fact that different assumptions on the star-formation history were made in different papers, most commonly the continuous star formation over a some time interval was assumed. In particular, relations of Kennicutt (1998) are obtained for a period of $\sim 100 \mathrm{Myr}$ for a star formation. We also note that adopting the IMF by Kroupa (2001) instead 

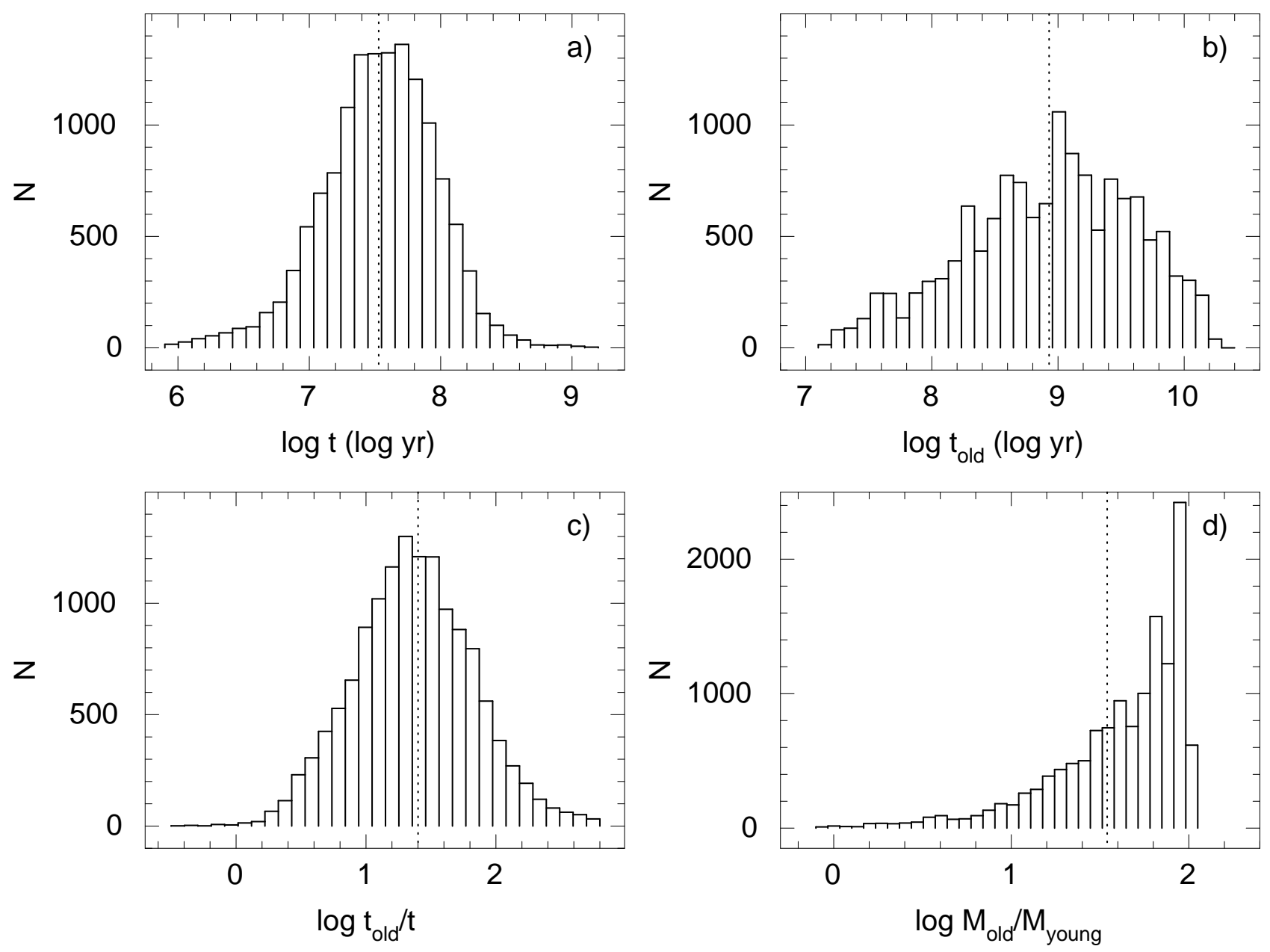

Fig. 6 Histograms of a) $t=M_{\star} / \mathrm{SFR}_{0}(\mathrm{H} \beta)$, b) the age of the oldest stars $t_{\text {old }}$ derived from SED fitting, c) $t_{\mathrm{old}} / t$, and $\mathbf{d}$ ) the mass ratio $M_{\text {old }} / M_{\text {young }}$ of the old stellar population with the age $>10$ Myr continuously formed with a constant SFR to the young stellar population formed in the burst of star formation with the age $<10$ Myr. $M_{\star}, M_{\text {young }}, M_{\text {old }}$ and $t_{\text {old }}$ are derived from SED fitting (Izotov et al. 2016a) 

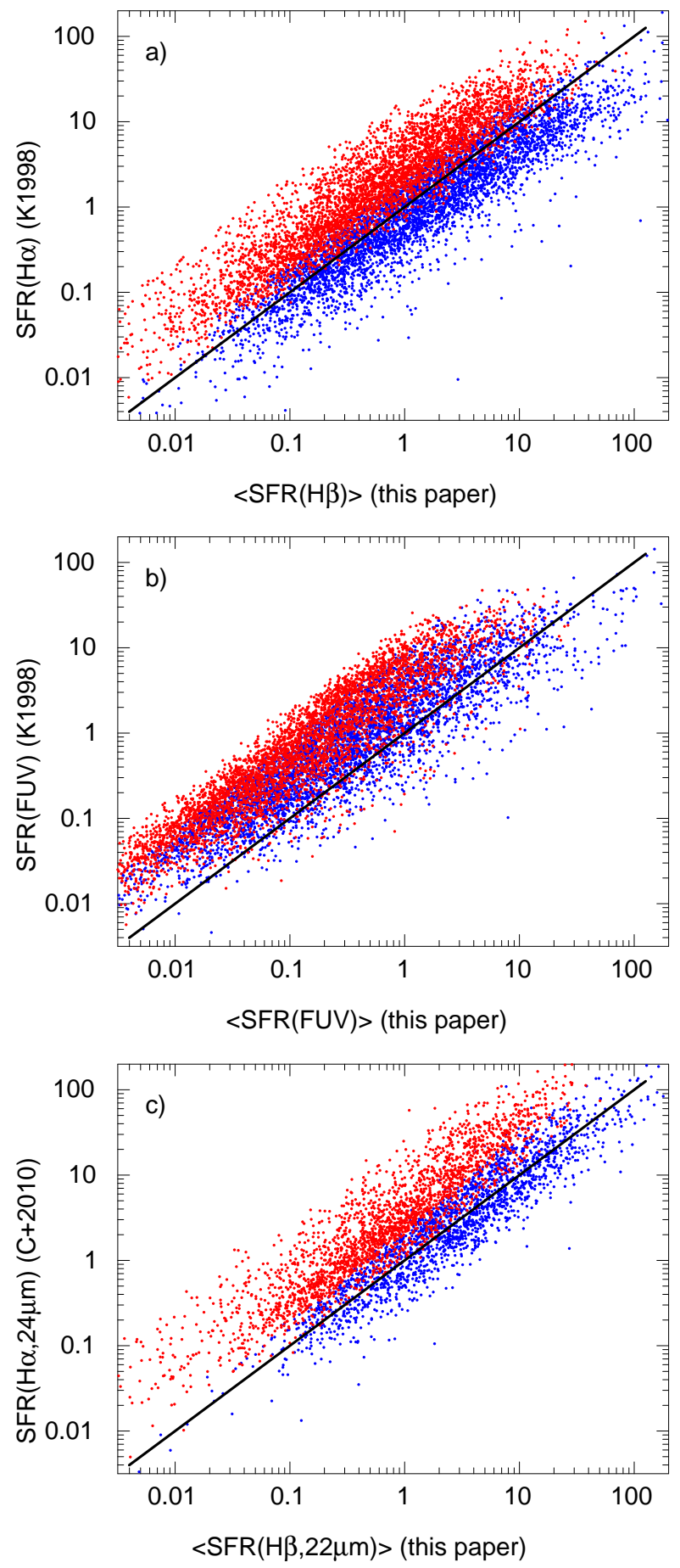

Fig. 7 The comparison of average star formation rates derived in this paper and SFRs obtained by other authors. Are shown relations between a) $<\mathrm{SFR}(\mathrm{H} \beta)>$ and $\operatorname{SFR}(\mathrm{H} \alpha)$ by Kennicutt (1998), b) $<\operatorname{SFR}(\mathrm{FUV})>$ and SFR(FUV) by Kennicutt (1998), and c) $<\operatorname{SFR}(\mathrm{H} \beta, 22 \mu \mathrm{m})>$ and $\operatorname{SFR}(\mathrm{H} \alpha, 24 \mu \mathrm{m})$ by Calzetti et al. (2010). All SFRs are expressed in $\mathrm{M}_{\odot} \mathrm{yr}^{-1}$. CSFGs with $\mathrm{EW}(\mathrm{H} \beta) \geq 50 \AA$ and $\mathrm{EW}(\mathrm{H} \beta)<50 \AA$ are shown by red and blue symbols, respectively. Solid lines in all panels are one by one relations of the Salpeter (1955) one would result in decrease of SFR by a factor of 1.44 (Kennicutt et al. 2009).

The determination of the interval of star formation in bursting CSFGs from our sample is somewhat uncertain. Therefore, the averaged SFR is strongly dependent on the value of this interval because of a rapid luminosity decline after the instantaneous burst. Furthermore, the star formation history prior the present starburst should somehow be taken into account.

To obtain the averaged SFR we first derive the time $t=M_{\star} / \mathrm{SFR}_{0}$ needed to build the mass of the galaxy with $\mathrm{SFR}_{0}$. The histogram of $\log t$ is shown in Fig. 6a. It is seen that $\log t$ is low and it varies in the relatively narrow range between 7 and 8 for most CSFGs from our sample ( $\sim 80$ percent) with the average value of 7.53 .

We compare $t$ with the age $t_{\text {old }}$ of the oldest stars formed in the CSFG. The latter quantity as well as $M_{\star}$ are derived from SED fitting of the SDSS spectra (Sect. 2. Izotov et al. 2016a). The distribution of $\log t_{\text {old }}$ is shown in Fig. 6 b. It is much broader than that of $\log t$ in Fig. 6a due to, in part, the dominating contribution of the young stellar population to the optical continuum in SDSS spectra. This contribution makes the determination of $t_{\text {old }}$ somewhat uncertain. From Fig. 6b we derive the average $\log t_{\text {old }}$ of 8.93 , which is much lower than the age of the Universe, indicating that most CSFGs from our sample were formed relatively recently, $\lesssim 1-2$ Gyr ago.

We define the star-formation rate $<\mathrm{SFR}>$ averaged over the galaxy lifetime for our bursting CSFGs according to the relation

$<\mathrm{SFR}>=\mathrm{SFR}_{0} / a$,

where $a=t_{\text {old }} / t$.

The distribution of $\log \left(t_{\text {old }} / t\right)$ is shown in Fig. 6r. with the average value of 1.4 , corresponding to $a=$ 25. It is interesting to compare this distribution with the mass ratio $M_{\text {old }} / M_{\text {young }}$ distribution of the stellar population formed during a recent starburst, and an older population with the age $>10$ Myr, continuously formed with a constant SFR (Fig. 6 d). We find that the average $\log \left(M_{\text {old }} / M_{\text {young }}\right)$ is 1.54 , or is slightly higher than the average $\log \left(t_{\text {old }} / t\right)$, implying that, in the case of the bursting star formation, $\sim 25-35$ starbursts with similar strengths are needed during the CSFG lifetime to build up its stellar mass.

Thus, we find that $<\operatorname{SFR}(\mathrm{H} \beta)>\sim 0.04 \times \operatorname{SFR}_{0}(\mathrm{H} \beta)$. Correspondingly, to keep SFRs derived from other star-formation indicators in different wavelength ranges equal to each other, we use the same factor 0.04 to convert respective $\mathrm{SFR}_{0} \mathrm{~s}$ at a zero starburst age to SFRs averaged over the galaxy lifetime. 
In Fig. 7 we compare the $<$ SFR $>$ s for CSFGs from our sample as defined by Eq. 8 with SFRs proposed by Kennicutt (1998) and Calzetti et al. (2010) by using various star-formation indicators obtained for the continuous star formation during the interval of $\sim$ 100 Myr. Fig. 77 represents the relation between the $<\mathrm{SFR}(\mathrm{H} \beta)>$ and $\operatorname{SFR}(\mathrm{H} \alpha)$ obtained by using the observed $\mathrm{H} \alpha$ luminosity and the relation by Kennicutt (1998). It is seen that SFR derived from the Kennicutt (1998) relation is $\sim 2$ times higher than the average SFR derived in this paper. The difference is higher for CSFGs with younger starbursts (red symbols) as evidenced by their high $\mathrm{EW}(\mathrm{H} \beta) \geq 50 \AA$. Similarly, the $<\mathrm{SFR}(\mathrm{FUV})>$ in Fig. $7 \mathrm{~b}$ is $\sim 3$ times lower than the SFR(FUV) obtained from the observed FUV luminosity and using the relation by Kennicutt (1998).

It was suggested in several papers that the combination of star formation indicators, which use both the UV and IR observed luminosities, are more reliable as compared to those which use a single star formation indicator. To check this, we compare $\operatorname{SFR}(\mathrm{H} \alpha, 24 \mu \mathrm{m})$ by Calzetti et al. (2010) with the use of the observed $\mathrm{H} \alpha$ and Spitzer $24 \mu \mathrm{m}$ luminosities, and $<\operatorname{SFR}(\mathrm{H} \beta, 22 \mu \mathrm{m})>$ as defined by Eqs. 7 and 8 . Similarly to Figs. 7a, the SFR with the Calzetti et al. (2010) relation is $\sim 2$ times higher than the $<\mathrm{SFR}>$ derived in this paper. We also note that there is an offset between the galaxies with high and low $\mathrm{EW}(\mathrm{H} \beta) \mathrm{s}$. This is because the bursting nature of star formation is not taken into account in the Kennicutt (1998) and Calzetti et al. (2010) relations, which use the observed luminosities instead of the luminosities reduced to a zero starburst age. On the other hand, we note that SFR calibrations by Kennicutt (1998) and Calzetti et al. (2010) can be used for approximate estimates within a factor of $\sim 2$ of the SFR in bursting galaxies averaged over their lifetime. These estimates would be useful for the determination of global cosmic chemical enrichment and the intensity of intergalactic radiation.

\section{Conclusions}

In this paper we discuss the relations for the determination of the star formation rate (SFR) in a large sample of $~ 14000$ bursting compact star-forming galaxies (CSFGs) selected by Izotov et al. (2016a) from the Data Release 12 (DR12) of the Sloan Digital Sky Survey (SDSS). These data are supplemented by the GALEX and WISE photometric data in the farultraviolet (FUV) and mid-infrared (MIR) ranges, respectively. We argue that the bursting nature of star formation in these galaxies casts doubts about applicability of commonly used relations for the determination of SFR (e.g. Kennicut 1998; Calzetti et al. 2010). This difference in the star-formation history is taken into account in this paper. Our main results are as follows.

1. We obtain relations for the determination of $\mathrm{SFR}_{0} \mathrm{~S}$ at a zero starburst age from the apertureand extinction-corrected $\mathrm{H} \beta$ luminosities, extinctioncorrected FUV luminosities, and observed $22 \mu \mathrm{m}$ luminosities, which are all reduced to a zero starburst age. We find the tightest relation between $\operatorname{SFR}_{0}(\mathrm{H} \beta)$ and a geometric mean of star formation rates in the FUV and at $22 \mu \mathrm{m}, \mathrm{SFR}_{0}(\mathrm{FUV}, 22 \mu \mathrm{m})$, while the relations between $\mathrm{SFR}_{0}(\mathrm{H} \beta)$ and $\mathrm{SFR}_{0}(\mathrm{FUV})$, and $\mathrm{SFR}_{0}(\mathrm{H} \beta)$ and $\mathrm{SFR}_{0}(22 \mu \mathrm{m})$ have considerably higher dispersions. We attribute these differences to statistical uncertainties in FUV and $22 \mu \mathrm{m}$ luminosities.

2. Using $\mathrm{SFR}_{0} \mathrm{~s}$ and results of SED fitting of the SDSS spectra we search for the recipe to estimate the star formation rate $<\mathrm{SFR}>$ averaged over the lifetime of the galaxy. We propose to use the relation $<\mathrm{SFR}>$ $=\mathrm{SFR}_{0} / a$ adopting $a=t_{\text {old }} / t$, where $t_{\text {old }}$ is the age of the oldest stars and $t$ is a time needed to build a stellar mass of the galaxy $M_{\star}$ with a constant star formation rate $\mathrm{SFR}_{0}$. The average value of $a$ is 25 and therefore $<\mathrm{SFR}>=0.04 \times \mathrm{SFR}_{0}$.

3. We compared relations between $<\mathrm{SFR}>\mathrm{s}$ and commonly used SFRs by Kennicutt (1998) and Calzetti et al. (2010) for our sample of galaxies and found that former ones are $\sim 2-3$ lower. Our relations for the SFR determination are likely more appropriate for CSFGs because they take into account rapid temporal evolution of their luminosities. However, the commonly used SFR calibrations can also be applied for approximate estimates within a factor about 2 of the SFR averaged over the lifetime of the bursting galaxy.

\section{Acknowledgments}

We thank the referee whose useful comments helped to make the paper clearer. SDSS-III has been provided by the Alfred P. Sloan Foundation, the Participating Institutions, the National Science Foundation, and the U.S. Department of Energy Office of Science. The SDSS-III web site is http://www.sdss3.org/. SDSS-III is managed by the Astrophysical Research Consortium for the Participating Institutions of the SDSS-III Collaboration. GALEX is a NASA mission managed by the Jet Propulsion Laboratory. This publication makes use of data products from the Wide-field Infrared Survey Explorer, which is a joint project of the University of California, Los Angeles, and the Jet Propulsion Laboratory, California Institute of Technology, funded by the National Aeronautics and Space Administration. 


\section{References}

Alam, S. et al. 2015, Astrophys. J. Suppl. Ser., 219, 12

Aller, L. H. 1984, Physics of Thermal Gaseous Nebulae (Dordrecht: Reidel)

Battisti, A. J., Calzetti, D., Johnson, B. D., Elbaz D. 2015, Astrophys. J., 800, id.143

Boquien, M. et al. 2016, Astron. Astrophys., 591, A6

Calzetti, D. et al. 2010, Astrophys. J., 714, 1256

Cardelli, J. A., Clayton, G. C., Mathis, J. S. 1989, Astrophys. J., 345, 245

Curtis-Lake, E. et al. 2013, Mon. Not. R. Astron. Soc., 429, 302

Draine, B. T., Li, A. 2007, Astrophys. J., 657, 810

Duarte Puertas, S. et al. 2017, Astron. Astrophys., 599, A71

Girardi, L., Bressan, A., Bertelli, G., Chiosi, C. 2000, Astron. Astrophys. Suppl. Ser., 141, 371

Hao, C.-N. et al. 2011, Astrophys. J., 741, 124

Hunt, L. K., Thuan, T. X., Izotov, Y. I., Sauvage, M. 2010, Astrophys. J., 712, 164

Izotov, Y. I., Thuan, T. X., Lipovetsky, V. A. 1994, Astrophys. J., 435, 647

Izotov, Y. I., Guseva, N. G., Fricke, K. J., Henkel, C. 2014 Astron. Astrophys., 561, A33

Izotov, Y. I., Guseva, N. G., Fricke, K. J., Henkel, C. 2016a, Mon. Not. R. Astron. Soc., 462, 4427

Izotov, Y. I. et al. 2016b, Mon. Not. R. Astron. Soc., 461, 3683

Izotov, Y. I. et al. 2018, Mon. Not. R. Astron. Soc., 474, 4514

Jiang, L. et al. 2013, Astrophys. J., 772, id.99

Karman, V. et al. 2017, Astron. Astrophys., 599, A28

Kashino, D. et al. 2013, Astrophys. J., 777, L8

Kennicutt, R. C. Jr. 1998, Annu. Rev. Astron. Astrophys., 36,189

Kennicutt, R. C. Jr. et al. 2009, Astrophys. J., 703, 1672

Kroupa, P. 2001, Mon. Not. R. Astron. Soc., 322, 231

Lee, J. C., Hwang, H. S., Ko, J. 2013, Astrophys. J., 774, id. 62

Leitherer, C. et al. 1999, Astrophys. J. Suppl. Ser., 123, 3

Leitherer, C. et al. 2014, Astrophys. J. Suppl. Ser., 212, 14

Lejeune, T., Buser, R., Cuisinier, F. 1997, Astron. Astrophys. Suppl. Ser., 125, 229

Panella, M. et al. 2015, Astrophys. J., 807, id. 141

Planck Collaboration XVI 2014, Astron. Astrophys., 571, A16

Puglisi, A. et al. 2016, Astron. Astrophys., 583, id. A83

Reddy, N. A. et al. 2010, Astrophys. J., 712, 1070

Reddy, N. A. et al. 2015, Astrophys. J., 806, id. 259

Salpeter, E. E. 1955, Astrophys. J., 121, 161

Schmutz, W., Leitherer, C., Gruenwald, R. 1992, Publ. Astron. Soc. Pac., 104, 1164

Shivaei, I., Reddy, N. A., Steidel, C. C., Shapley, A. E. 2015, Astrophys. J., 804, id.149

Shivaei, I. et al. 2016, Astrophys. J., 820, L23

Weisz, D. R. 2011, Astrophys. J., 739, 5

Wright, E. L. 2006, Publ. Astron. Soc. Pac., 118, 1711

Wu, Y., Charmandaris, V., Hao, L., Brandl, B. R., BernardSalas, J., Spoon, H. W. W., Houck, J. R. 2006, Astrophys. J., 639, 157

Yang, H. et al. 2017, Astrophys. J., 844, 171

Zhang, F., Li, L., Kang, X., Zhuang, Y., Han, Z. 2013, Mon. Not. R. Astron. Soc., 433, 1039

This manuscript was prepared with the AAS LATEX macros v5.2. 